\title{
Suspended photonic waveguide arrays for submicrometer alignment
}

\author{
Tjitte-Jelte Peters, Marcel Tichem and Urs Staufer \\ Micro \& Nano Engineering Lab, Delft University of Technology, Mekelweg 2, Delft, Netherlands
}

\begin{abstract}
This paper presents a new alignment concept for the alignment of multichannel photonic intergrated circuits (PICs) using flexible photonic waveguides on one of the PICs that are positionable by integrated micro electro mechanical system (MEMS) actuators. The concept aims for high precision and high degree of assembly process automation. The proposed concept includes pre-alignment of both PICs on a common substrate followed by fine-alignment using the on-chip flexible waveguides and MEMS functionality. This paper introduces the alignment approach and reports on the development and fabrication of suspended and mechanically flexible photonic waveguides.

Single suspended waveguide beams and suspended arrays with two and four coupled parallel waveguide beams of different lengths ( $250 \mu \mathrm{m}$ to $1000 \mu \mathrm{m})$ and different widths $(18 \mu \mathrm{m}$ to $34 \mu \mathrm{m})$ are designed and fabricated. After fabrication, waveguide beam fracturing is observed. The fabrication process has been extended by an additional under-etching step in order to reduce beam fracturing. The static out-of-plane deflection of the fabricated devices follows a specific profile with a dominating upward curvature resulting in a measured maximum out-of-plane deflection of $2 \%$ of the length. The beam stiffness of the fabricated devices is measured and proves to be within the available force of microactuators.
\end{abstract}

\section{INTRODUCTION}

The continuous development of photonic technology leads to new kinds of multiport PICs for use in the fields of telecom, datacom, optical sensing and visible light applications. The advances in packaging of complex PICs are lagging behind, making packaging a large contributor to fabrication costs. ${ }^{1}$ Moreover, the complexity of packaging PICs increases with the number of optical ports. Improvement in optical packaging and interconnect technology is of great importance for the future of PICs.

Within the packaging process, alignment of photonic components is a crucial step. Two categories of alignment approaches can be distinguished: active and passive alignment. Active alignment uses the measured light coupled through the optical path as a feedback signal in order to attain the position with lowest coupling loss. In passive alignment the final position is obtained without detecting the coupling of light. Usually passive alignment uses alignment structures or alignment marks. Once successfully aligned, another challenge is attachment without disturbing the alignment. For fixation mostly epoxy attachment, solder attachment or mechanical clamping is implemented. Depending on the attachment method, the alignment might be affected by a post-bonding shift.

Different implementations of alignment approaches have been demonstrated in literature, based on either active alignment, passive alignment or a hybrid form. For example, active alignment and clamping of an optical fibre is achieved using microelectromechanical actuators. ${ }^{2}$ Passive alignment with submicrometer accuracy is reported using two photonic chips on a silicon optical bench. ${ }^{3}$ Furthermore, alignment of multi-channel configurations is studied utilizing a micromechanical connector, ${ }^{4}$ a grating coupler array, ${ }^{5}$ laser adjustment ${ }^{6}$ and spot size converters (SSCs). ${ }^{7}$ Current technology for precise photonic assembly employs the use of micromanipulators. ${ }^{8}$

Passive photonic alignment is usually a much preferred method over traditional active photonic alignment, because the latter can be time-consuming and costly. ${ }^{9,10}$ In this paper we present a new concept using MEMS technology to enable high-precision, fast and affordable photonic active alignment of a multiport PIC with a fibre array. The proposed alignment concept comprises a two-step sequence: coarse pre-alignment followed by precise fine-alignment using MEMS functionality.

The aim of this paper is to report on the fabrication feasibility of suspended and mechanically flexible photonic waveguides to be used within the high precision alignment step. The design, the fabrication and the characterization of suspended waveguide structures are presented in this document. First the proposed overall alignment concept is explained in more detail (Section 2). Next, the fabrication method is presented (Section 3). The fabrication results are presented in Section 4 and discussed in Section 5. Section 6 contains the conclusions.

Silicon Photonics and Photonic Integrated Circuits IV, edited by Laurent Vivien, Seppo Honkanen,

Lorenzo Pavesi, Stefano Pelli, Proc. of SPIE Vol. 9133, 913317 · (c) 2014 SPIE

CCC code: $0277-786 X / 14 / \$ 18 \cdot$ doi: $10.1117 / 12.2052430$

Proc. of SPIE Vol. $9133913317-1$ 

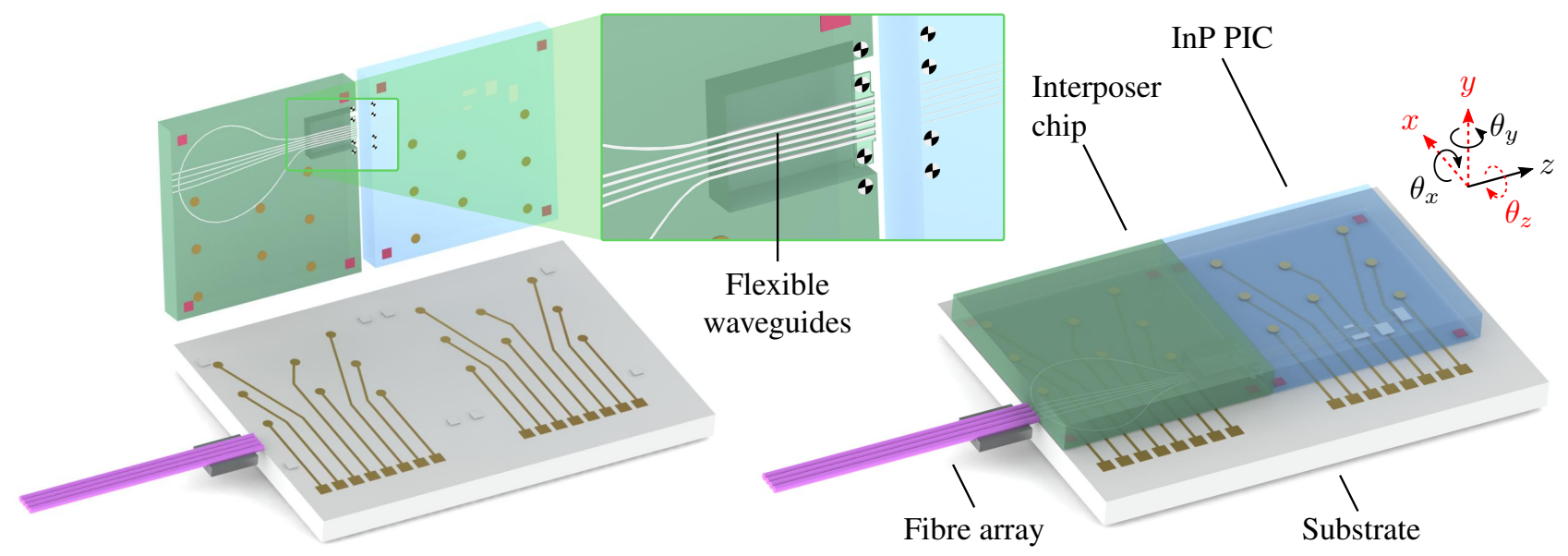

Figure 1: Schematic representation of pre-alignment of the assembly. Left: before pre-alignment. The inset shows a closeup view of the partly suspended and flexible waveguide array. Right: the interposer chip and PIC are flip-chip mounted on the substrate. The DOFs aligned within pre-alignment are indicated in black (solid) and the DOFs to be aligned in fine-alignment are indicated in red (dashed)

\section{ALIGNMENT APPROACH}

The proposed alignment concept offers high precision alignment of two PICs by using microfabricated functionality and mechanically flexible waveguides, both integrated in one of the PICs. In relation to comparable alignment approaches our alignment concept aims to reach higher accuracy and precision while reducing the time of alignment. The proposed concept offers alignment of waveguide arrays making it suitable for multiport PICs. The increase in fabrication costs is limited because most of the functionality is realized on the interposer chip through post-processing, for the most part using existing chip area that otherwise remains unused. Moreover, standard microfabrication processes suffice for the realization of these functions in the post-processing step. The alignment concept is designed for increased automation capability to ensure low cost and short assembly times.

Indium phosphide (InP) based technology is a leading candidate for complex (multiport) photonic circuits. ${ }^{1}$ If a fibre array is butt coupled to a multiport InP chip, the difference in mode field diameter (MFD) of the InP waveguides and the fibres causes significant loss of the optical signal. In theory, this problem can be solved by implementing SSCs, but at this moment it is technically very challenging to realize SSCs in InP technology which enable butt coupling to a fibre array with acceptable coupling efficiency. Our alignment approach combines InP-based technology with TriPleX platform technology to enable sufficiently low coupling loss of the optical signal. The TriPleX platform technology offers buried dielectric waveguide structures with low loss through the full wavelength range from visible to infrared. ${ }^{11,12}$ An advantage of this technology is the ability to design and realize both in-plane and out-of-plane SSCs.

Three benefits result from positioning an interposer chip based on TriPleX technology between a fibre array and an InP chip. Firstly, the coupling losses are reduced by minimizing the difference in MFD at both interfaces of the interposer chip. Secondly, when a fan-out of the waveguides is implemented on the interposer chip, the pitch of the InP waveguides can be smaller than the fibre pitch, reducing required chip-area and thereby lowering costs. Finally, combining different material technologies in a hybrid package allows implementing additional functionality, and rethinking functional partitioning between the PICs, in order to benefit most from each material platform's characteristics.

While the optical waveguides on the interposer chip are passive so have no need for electrical connections, the microactuators and possibly the fixing mechanism do require some electronic control. In our alignment approach, solder bump technique is utilized to get the interposer chip both electrically and mechanically connected to the substrate. The InP chip is attached in the same way, creating an electrical connection to power the light transmitter and receiver during the active fine-alignment step.

Submicrometer alignment is achieved in two steps. First, the InP chip and interposer chip are coarsely pre-aligned and then attached to a common substrate (see Figure 1). At this stage an alignment precision around $5 \mu \mathrm{m}$ is sufficient. 
Passive alignment structures in combination with flip-chip bonding technology are used to align and mount the chips on the substrate, which is a fast and cost-effective technique offering the pre-alignment accuracy necessary for the subsequent fine-alignment. Second, microactuators fine-adjust the position of the flexible waveguide array to obtain maximum lightcoupling and finally the waveguide array is fixed in its final position. The goal is a final misalignment smaller than $300 \mathrm{~nm}$. To illustrate the principle of fine adjustment, alignment in the $x$-direction only is presented in Figure 2.

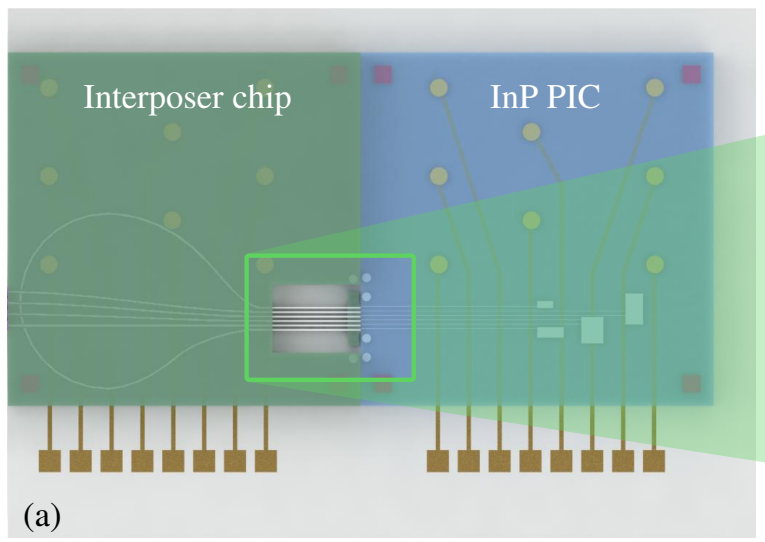

Flexible waveguides Crossbar

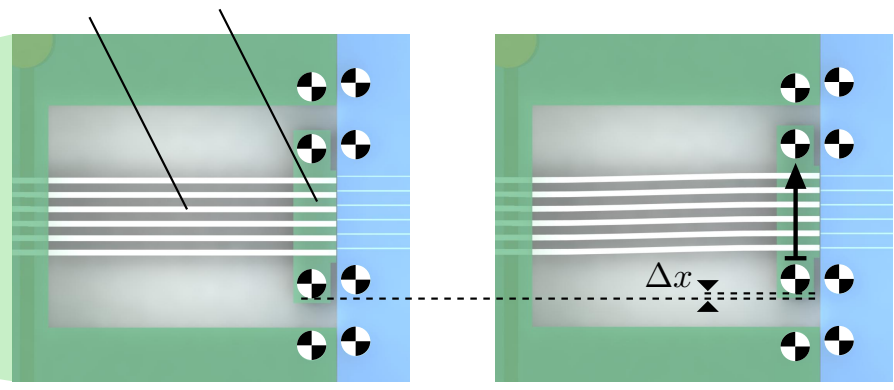

(b)

(c)

Figure 2: Schematic representation (top view) of in-plane fine alignment and fixation of the waveguides. (a) Top view of the interposer chip and PIC after coarse pre-alignment. (b) Close-up view of the flexible part of the waveguides before fine alignment. A small misalignment of the waveguides and alignment marks is visible. (c) Close-up view of the flexible part of the waveguides after fine-alignment. Fine-alignment is achieved by translating the crossbar over a distance $x$ in the direction of the arrow (no microactuator is included in this schematic representation)

Since the pitch of the interposer waveguides is defined by photolithography, it can be very accurately realized to be similar to that of the InP waveguides. In order to retain this well defined pitch, the design includes a rigid crossbar located at the free end of the interposer waveguide array. Furthermore, the number of required actuators is significantly reduced by employing a waveguide array with a crossbar. For accurate displacement of this crossbar in the fine-alignment step, microactuators are integrated in the interposer chip.

The concept requires three out of six degrees of freedom (DOFs) to be sufficiently aligned after the pre-alignment: $z, \theta_{\mathrm{x}}$ and $\theta_{\mathrm{y}}$ (the definition of the coordinate system used in this paper is shown in Figure 1). In terms of coupling efficiency the alignment demands for the $z$-translation and $\theta_{\mathrm{x}}$-rotation are lower than the demands for the other DOFs. Additionally, the requirements in $x$ and $\theta_{\mathrm{y}}$ are relaxed by in-plane SSCs at the interposer interface of the InP waveguides. This means that after pre-alignment the most critical DOFs for low coupling loss are $x, y$ and $\theta_{\mathrm{z}}$. Consequently, the microactuators must be capable of displacement in $x$-direction, $y$-direction and rotation around the $z$-axis. An obvious but important requirement is for the microactuator-strokes to be larger than the pre-alignment error in the corresponding DOF.

This paper reports on the first results of developing the flexible waveguide function.

\section{TECHNOLOGY AND FABRICATION OF SUSPENDED WAVEGUIDES}

This section explains the fabrication process of the flexible waveguide structures. First the waveguide technology is introduced in Section 3.1. In Section 3.2 the fabrication process of buried waveguides is presented and Section 3.3 provides the process details for the release of buried waveguides to make them mechanically flexible in the alignment area.

\subsection{Waveguide technology}

The suspended waveguides are based on TriPleX technology and are created by selectively removing material around buried waveguides. The basic concept of a TriPleX waveguide is a stoichiometric silicon nitride $\left(\mathrm{Si}_{3} \mathrm{~N}_{4}\right)$ core encapsulated in a silicon dioxide $\left(\mathrm{SiO}_{2}\right)$ cladding. A cladding thickness of $8 \mu \mathrm{m}$ is used to reduce propagation loss as a result of scattering at the $\mathrm{SiO}_{2}$-air and $\mathrm{SiO}_{2}$-silicon interface. Different geometries can be realized, varying from single stripes to box shape waveguides. ${ }^{11}$ The more advanced box shape geometry provides the means for in-plane and out-of-plane spot 
size conversion. ${ }^{13}$ But since the focus of this paper is on suspended waveguides, the single stripe geometry is used in order to simplify fabrication of the waveguides.

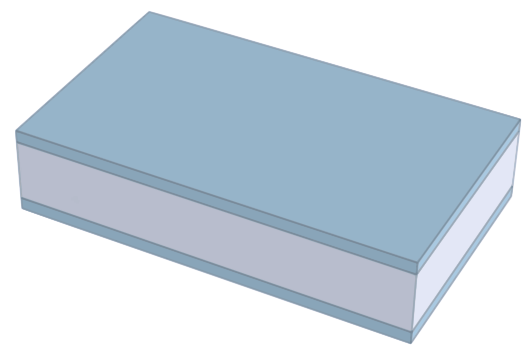

(a)

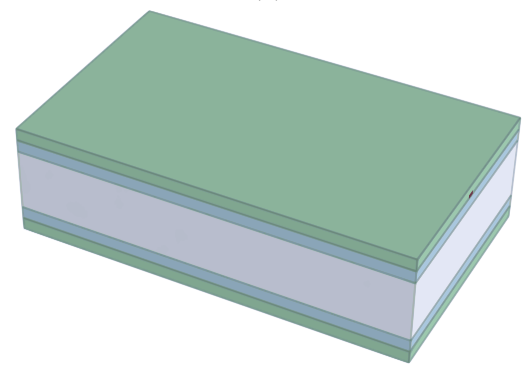

(d)

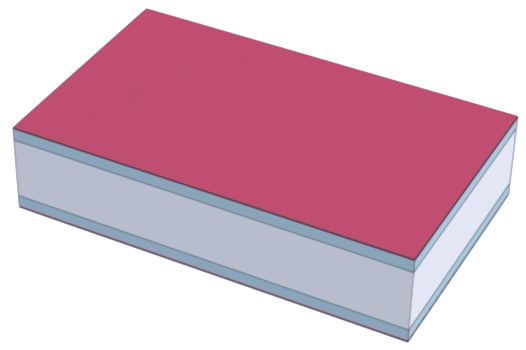

(b)

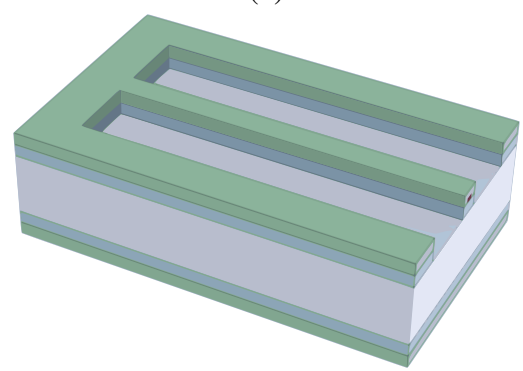

(e)

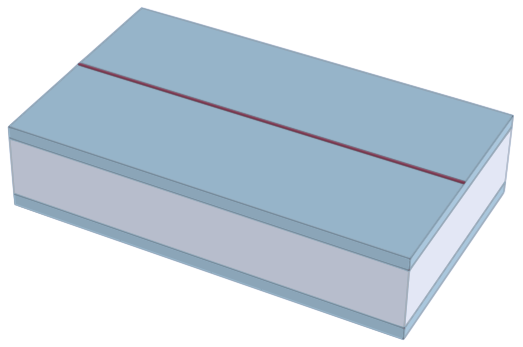

(c)

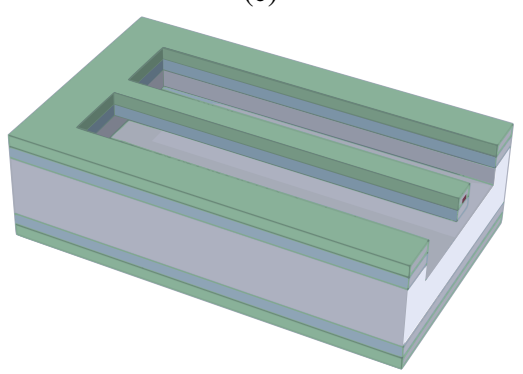

(f)

Figure 3: Fabrication process of a single suspended waveguide: (a) Thermal oxidation (b) LPCVD of $\mathrm{Si}_{3} \mathrm{~N}_{4}$ (c) Frontside and backside $\mathrm{RIE}$ of $\mathrm{Si}_{3} \mathrm{~N}_{4}$ (d) Frontside and backside PECVD of $\mathrm{SiO}_{2}$ followed by thermal annealing. Now the fabrication of a buried waveguide is finished (e) Plasma etching of $\mathrm{SiO}_{2}$ (f) Plasma (under)etching of $\mathrm{Si}$

\subsection{Fabrication of buried waveguides}

Fabrication consists of deposition of the different layers for the bottom cladding, waveguide core and top cladding and patterning of the waveguide core. The first step is thermal oxidation of a silicon ( $\mathrm{Si}$ ) wafer to form an $8 \mu \mathrm{m}$ thick $\mathrm{SiO}_{2}$ bottom cladding layer (Figure 3a). A $220 \mathrm{~nm}$ thin layer of $\mathrm{Si}_{3} \mathrm{~N}_{4}$ is deposited on top of the thermal $\mathrm{SiO}_{2}$ layer by a low pressure chemical vapour deposition (LPCVD) process (Figure 3b). After adding and patterning a photoresist layer, this pattern is transferred into the $\mathrm{Si}_{3} \mathrm{~N}_{4}$ layer (Figure 3c) using a reactive ion etch (RIE) process with $\mathrm{CF}_{4}, \mathrm{SF}_{6}$ and $\mathrm{O}_{2}$ gasses. The resulting $2 \mu \mathrm{m}$ wide stripes will function as waveguide cores. For the top cladding, an $8 \mu \mathrm{m}$ thick layer of $\mathrm{SiO}_{2}$ is deposited by plasma enhanced chemical vapour deposition (PECVD) (Figure 3d) and annealed for three hours at a temperature of $1100^{\circ} \mathrm{C}$. A limited change in temperature is obtained by a ramp-up and ramp-down of $10^{\circ} \mathrm{C} \mathrm{min}^{-1}$ to prevent cracking of the $\mathrm{PECVD} \mathrm{SiO}_{2}$ layer.

\subsection{Release of buried waveguides}

Release of the waveguides is done by selectively etching through the $\mathrm{SiO}_{2}$ layer followed by under-etching of the Si layer. First a $6 \mu \mathrm{m}$ thick photoresist layer is applied on top of the PECVD $\mathrm{SiO}_{2}$ and patterned. The design of the photolithography mask used in this step allows control over dimensional parameters like the width and the length of the suspended waveguide-beam. For the $\mathrm{SiO}_{2}$ etch an RIE process is used with $\mathrm{CF}_{4}$ and $\mathrm{CHF}_{3}$ gasses, stopping on the $\mathrm{Si}$ wafer. In order to release specific waveguide beams from the $\mathrm{Si}$, two sequential dry etch processes are used. The $\mathrm{Si}$ is first anisotropically etched and then isotropically etched. For the anisotropic etching an inductively coupled plasma (ICP) etch is used with $\mathrm{SF}_{6}$ and $\mathrm{O}_{2}$ gasses at a flow rate of $20 \mathrm{sccm}$, a process pressure of $50 \mathrm{~m}$ Torr and a wafer temperature of $10^{\circ} \mathrm{C}$. The isotropic etch is performed by means of an ICP etch with $\mathrm{SF}_{6}$ gass at a flow rate of $30 \mathrm{sccm}$, a process pressure of $50 \mathrm{mTorr}$ and a wafer temperature of $20^{\circ} \mathrm{C}$. 
Table 1: Measured dimensions of fabricated waveguide beams

\begin{tabular}{lcccc}
\hline & Designed $(\mu \mathrm{m})$ & \multicolumn{3}{c}{ Measured $(\mu \mathrm{m})$} \\
\hline Thickness & 16 & & & 15.1 \\
Length (L) & 250 & & & 247 \\
& 500 & & & 498 \\
& 750 & & & 743 \\
& 1000 & top & bottom & average \\
& & 12.4 & 18.5 & 15.45 \\
Width (W) & 18 & 20.7 & 26.6 & 23.65 \\
& 26 & 27.6 & 34.8 & 31.2 \\
Undercut (U) & 34 & & & $20-25$ \\
\hline
\end{tabular}

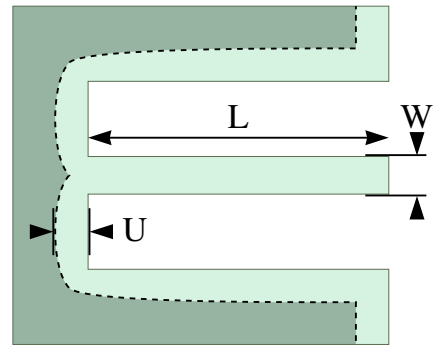

Figure 4: Schematic representation (top view) of a single cantilever waveguide beam showing the beam length (L), the beam width (W) and the undercut (U)

\section{RESULTS}

This section presents the results of microfabrication of diverse waveguide structures and the mechanical characterisation, which includes quantifying static beam deflection and bending stiffness.

\subsection{Microfabrication results}

The fabrication results of three designs will be discussed in this section: single cantilever waveguide beams, suspended arrays of two waveguide beams and suspended arrays of four waveguide beams. We define a suspended array as a number of parallel cantilever waveguide beams, at their free ends connected by a crossbar. Varying the waveguide beam width $(18 \mu \mathrm{m}$, $26 \mu \mathrm{m}$ and $34 \mu \mathrm{m})$ and length $(250 \mu \mathrm{m}, 500 \mu \mathrm{m}, 750 \mu \mathrm{m}$ and $1000 \mu \mathrm{m})$ resulted in size variations of all three designs. In the designs with suspended arrays, the width of the crossbar is twice the waveguide beam width. The designed waveguide beam thickness is $16 \mu \mathrm{m}$. Every design is both realized with and without $\mathrm{Si}_{3} \mathrm{~N}_{4}$ core in the centre of the waveguide beams to quantify the effect of the presence of the $\mathrm{Si}_{3} \mathrm{~N}_{4}$ core on mechanical behaviour.

The designed and measured dimensions of the fabricated beams are presented in Table 1. The thickness is measured using white light interferometric measurements and all other beam dimensions are measured using scanning electron micrographs. Due to erosion of the photoresist mask during the $\mathrm{SiO}_{2}$ etching, the width of the beams is smaller at the top surface than at the bottom surface resulting in a trapezoidal cross-section. The corresponding rectangular width (equivalent to that of a rectangular beam) is approximately $8 \%$ to $14 \%$ smaller than the designed width.

In order to release the waveguide beams, the underlying silicon is removed by under-etching. In this etching step, the $\mathrm{SiO}_{2}$ layer can be considered as a mask: the silicon is etched while the $\mathrm{SiO}_{2}$ is not. The isotropic under-etching of silicon starts at every edge of the $\mathrm{SiO}_{2}$ mask. As a consequence, the waveguide beams are not only released, but also experience an effective increase in length due to the under-etching: at the base the cantilevers are extended by an overhanging membrane. This effect is visualized in Figure 4. The amount of undercut is optically measured and ranges from $20 \mu \mathrm{m}$ to $25 \mu \mathrm{m}$.

The under-etching of the silicon in order to release the $\mathrm{SiO}_{2}$ beam structures resulted in beams fracturing and snapping off. Figure 5 shows the yield of cantilever beams having a constant cross section (width of $14 \mu \mathrm{m}$ and thickness of $15.1 \mu \mathrm{m}$ ) and a length varying from $10 \mu \mathrm{m}$ to $1000 \mu \mathrm{m}$ on a single wafer with 36 dies. These results show release without fracturing up to a length of approximately $400 \mu \mathrm{m}$. Beyond this length, cantilevers are observed to be either intact or snapped. In case of snapping, a part of the cantilever remains at the base and the fracture position along the cantilever seems to be random. Cantilevers without $\mathrm{Si}_{3} \mathrm{~N}_{4}$ core show a slightly higher probability of fracturing than cantilevers with $\mathrm{Si}_{3} \mathrm{~N}_{4}$ core.

As with the single cantilevers, the release process of waveguide beam arrays resulted in snapped off beams. Figure 6 shows an array of four waveguide beams from which the two outer beams are snapped off. An explanation for the snapping of the beams might be found in the formation of a clamped-clamped beam situation during underetching. Because the waveguide beam width is smaller than the crossbar width, the waveguide beams are released before the crossbar is released. Until the crossbar is released, the waveguide beams are fixed on both sides. We have developed a two-step release process to prevent this situation. More information on the cause of beam fracturing and the two-step release process is given in Section 5. The proposed two-step release process resulted in more intact structures of all three designs. Figure 7 shows 


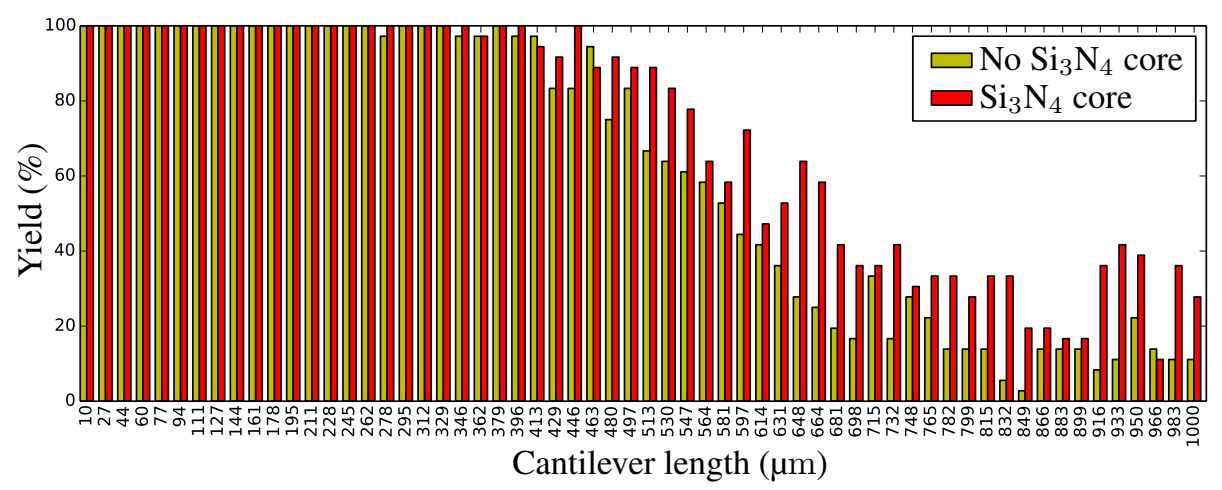

Figure 5: Yield of cantilevers with constant cross section (width of $14 \mu \mathrm{m}$ and thickness of $15.1 \mu \mathrm{m}$ ) plotted against cantilever length

SEM images of released single cantilever waveguide beams, an array of two waveguides and an array of four waveguides. All structures in Figure 7 are released using the two-step release method, except for the bottom single cantilever in Figure $7($ a).

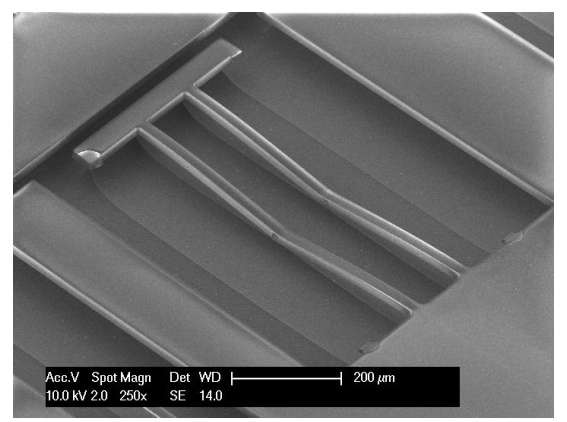

Figure 6: SEM image showing a damaged array of four waveguide beams as observed after the silicon etch

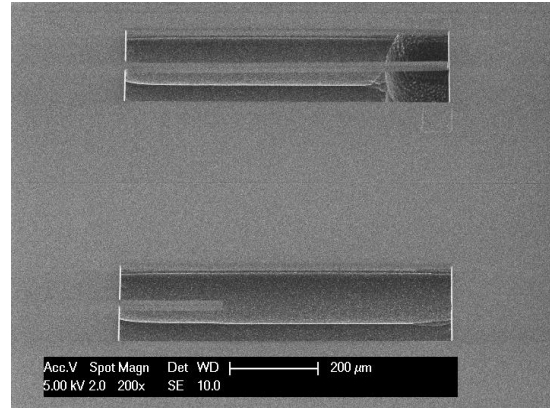

(a) Two single cantilevers

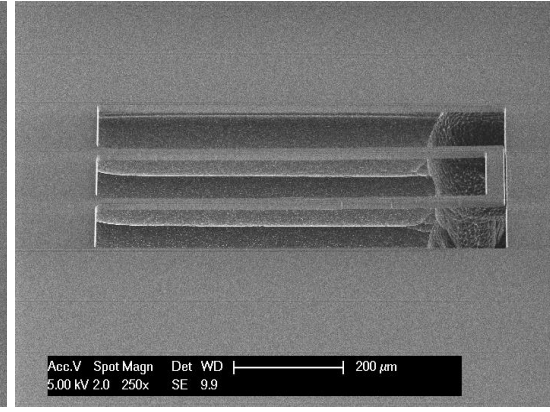

(b) Array of two waveguide beams

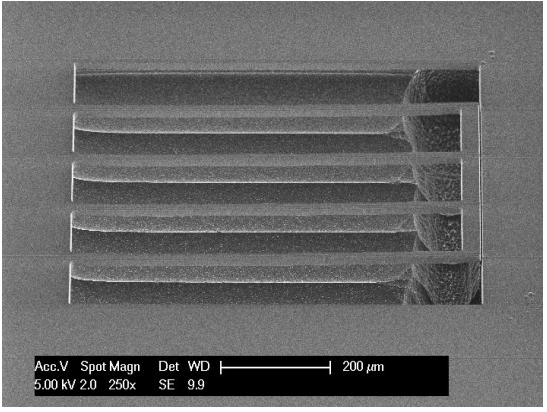

(c) Array of four waveguide beams

Figure 7: SEM images of released structures: all structures are released using the two-step release method, except for the bottom single cantilever in (a). Waveguide beams are $18 \mu \mathrm{m}$ wide and $750 \mu \mathrm{m}$ long. Figure 7 (a) shows one intact and one broken single cantilever, (b) shows an array of two suspended waveguide beams at their free ends connected by a crossbar and (c) shows an array of four suspended waveguide beams at their free ends connected by a crossbar

\subsection{Beam curvature}

The fabricated suspended waveguide beams show out-of-plane deformation. Profiles of the cantilever beams are measured using white light interferometric profiliometry and the static transverse deflection measurements are presented in Figure 8. 
Suspended beams with a particular width but different lengths follow the same profile. The cantilevers reveal an initial downward slope at the base of the cantilever. The suspended part from the base to the free end experiences upward bending with a constant radius of curvature. This profile leads to a deflection of the free end of $0 \mu \mathrm{m}, 3 \mu \mathrm{m}, 10 \mu \mathrm{m}$ and $20 \mu \mathrm{m}$ for the $250 \mu \mathrm{m}, 500 \mu \mathrm{m}, 750 \mu \mathrm{m}$ and $1000 \mu \mathrm{m}$ cantilever length respectively. The corresponding angular deflections of the free end are $0.3^{\circ}, 1.2^{\circ}, 1.9^{\circ}$ and $2.7^{\circ}$. The arrays of waveguide beams show similar out-of-plane bending behaviour.

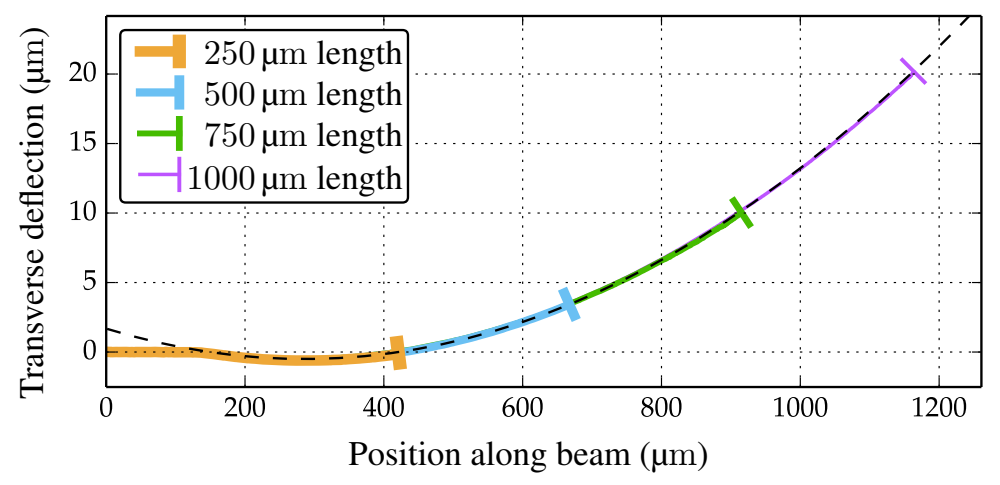

Figure 8: Static transverse deflections of fabricated cantilever beams measured by interferometric profilometry. The cantilever beams in this graph have a designed width of $18 \mu \mathrm{m}$. The dashed line indicates the outline of a circle with a $18.6 \mathrm{~mm}$ radius

\subsection{Beam stiffness}

The beam stiffness is determined using an estimate of the mass and the measured resonant frequency of the microbeams. Equation 1 shows the relation between resonant frequency $f_{\mathrm{n} 1}$, stiffness $k$ and effective mass $m_{\text {eff }}$ applicable for single cantilevers. The effective mass is $\frac{33}{140}$ times the mass of a cantilever. The cantilever mass is determined by the approximated volume (based on the measured dimensions) and the density. A $\mathrm{SiO}_{2}$ density of $2.2 \mathrm{~g} / \mathrm{cm}^{3}$ was assumed. Laser Doppler vibrometer measurements are performed to measure the resonant frequency of the beams. The experimental resonant frequency and stiffness of fabricated single beams and the theoretical stiffness are included in Table 2.

$$
f_{\mathrm{n} 1}=\frac{1}{2 \pi} \sqrt{\frac{k}{m_{\mathrm{eff}}}}
$$

Table 2: Experimental and theoretical out-of-plane bending stiffness of waveguide beams

\begin{tabular}{ccccc}
\hline Length $(\mu \mathrm{m})$ & Mass $(\mu \mathrm{g})$ & $\begin{array}{l}\text { Experimental } \\
\text { resonant frequency }(\mathrm{kHz})\end{array}$ & $\begin{array}{l}\text { Experimental } \\
\text { stiffness }\left(\mathrm{N} \mathrm{m}^{-1}\right)\end{array}$ & $\begin{array}{l}\text { Theoretical } \\
\text { stiffness }\left(\mathrm{N} \mathrm{m}^{-1}\right)\end{array}$ \\
\hline 498 & 0.256 & 54 & 6.94 & 7.54 \\
743 & 0.381 & 24 & 2.04 & 2.27 \\
988 & 0.507 & 14 & 0.92 & 0.97 \\
\hline
\end{tabular}

\section{DISCUSSION}

\subsection{Beam curvature}

Cantilever beams are known to deform out-of-plane due to relief of the residual stress following removal of the supporting material. Two components can be distinguished in the residual stress within (thermal) $\mathrm{SiO}_{2}$ : uniform and gradient stress. These components determine the profile of cantilever beams: the uniform stress component leads to axial elongation of a beam while the gradient stress component causes a beam to curl with a constant radius of curvature. ${ }^{14}$ 
If we look at the worst case (the profile of a $18 \mu \mathrm{m}$ wide and $1000 \mu \mathrm{m}$ long cantilever) the $20 \mu \mathrm{m}$ out-of-plane deflection of the free end can be considered as an out-of-plane offset. This out-of-plane offset can be solved by designing the distance between the common alignment substrate and the surface of the interposer chip accordingly. It must be noted that height compensation of the PICs to bring the waveguides nominally at the same level is required anyway, since the waveguides have a different distance with respect to the PIC's surface, regardless of any out-of-plane waveguide bending.

An interesting issue following from the beam curvature is related to the rotational misalignment. Suppose both the interposer chip and the InP chip are horizontally positioned so that the waveguide cores at the end facets are aligned. In this situation, a rotational misalignment between the waveguides will exist due to the curvature of the cantilever beam. Using the white light interferometric profile measurements, the radius of curvature is determined to be $\sim 18.6 \mathrm{~mm}$. Consequently, the free end of a $1000 \mu \mathrm{m}$ long beam will have an angular deflection (or rotational misalignment with a horizontal waveguide) of $2.7^{\circ}$. Depending on the MFD a rotational misalignment of this order can cause unacceptable loss. The out-of-plane microactuators that we are considering in our concept induce a deflection towards the backside of the chip. This means that the rotational misalignment caused by the curvature is reduced when the out-of-plane actuators are activated. Reducing the initial angular deflection can be achieved by minimizing the curl of the cantilever beam, for example by adding a stress-compensating layer on top of the beam.

The materials of the waveguide beams in this work differ from the original TriPleX technology. For the bottom cladding (thermal $\mathrm{SiO}_{2}$ ) exactly the same material as in TriPleX technology is used. The materials of the waveguide core $\left(\mathrm{Si}_{3} \mathrm{~N}_{4}\right)$ and top cladding (PECVD $\mathrm{SiO}_{2}$ ) correspond to the TriPleX materials, but they are deposited in a different facility. As a result, the material properties might differ from the TriPleX material properties. The curvature of suspended TriPleX waveguide beams will be investigated in prospective research.

\subsection{Beam stiffness}

The alignment concept uses integrated MEMS actuators to position the waveguide beams. MEMS actuators provide a limited force at a particular range. The stiffness of the flexible waveguide beams must be small enough to allow deflection by the limited force of MEMS actuators. Thermal actuators can deliver a maximum force in the millinewton range. Whether a suspended array of parallel waveguide beams is positionable by a thermal actuator depends on the number of beams and the beam dimensions. For example, a $20 \mu \mathrm{m}$ out-of-plane deflection of the free end of an array of four waveguide beams (each $18 \mu \mathrm{m}$ wide and $1000 \mu \mathrm{m}$ long) requires a force of approximately $74 \mu \mathrm{N}$. This value falls well within the capabilities of thermal actuators.

\subsection{Fracturing of beams}

It is observed that up to a certain length all fabricated waveguide beams are intact while beyond this length beams can fracture. Considering this length-dependent outcome, it is likely that the fracturing of waveguide beams has a mechanical cause. The exact mechanism is at this moment not fully understood.

As mentioned in Section 4.1, we have developed a two-step-release method to reduce the risk of beam fracturing. The idea of this method is to avoid clamped-clamped beam formation. In case of uniform stress, a clamped-clamped beam configuration is fundamentally different from that of a cantilever beam. On removal of the material supporting a $\mathrm{SiO}_{2}$ cantilever beam, uniform stress is relieved by axial elongation inducing vertical displacement of the free end. When the supporting material is removed underneath a clamped-clamped beam, complete stress relaxation through vertical displacement is impossible and instead the axial elongation leads to buckling of the beam. One can imagine that the stress concentrations in a clamped-clamped beam are higher than the stress concentrations in a cantilever beam.

The two-step-release method is visualized in Figure 9. Figure 9a and $9 \mathrm{~b}$ show the under-etching and release of the crossbar. During this etching step, the waveguide beams are protected by photoresist, except nearby the crossbar. When the crossbar is fully suspended, the photoresist is removed and the waveguide beams are under-etched (Figure 9c and 9d). Now that the crossbar is suspended, axial elongation can happen through vertical displacement of the crossbar.

\section{CONCLUSIONS}

The paper proposed a new concept for waveguide alignment of multiport photonic chips. The essence of the approach is to integrate MEMS-based alignment functionality on one of the chips. The approach potentially allows improved precision 


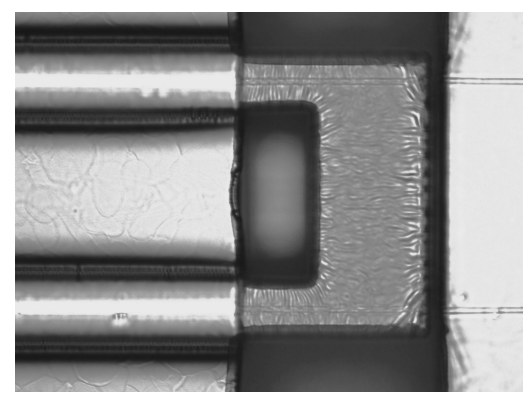

(a)

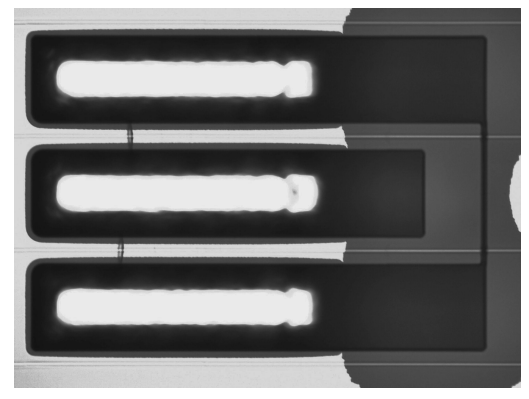

(c)

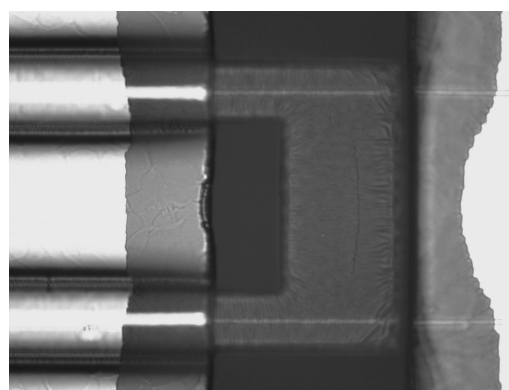

(b)

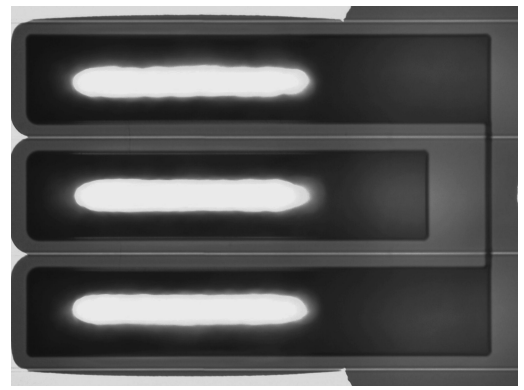

(d)

Figure 9: Microscopic images showing the two-step release of an array of two waveguide beams. In the first step the crossbar is released: (a) the crossbar is still attached after two minutes of isotropic etching, (b) the crossbar is fully suspended after 17 minutes of isotropic etching. The waveguide beams are released in the second step: (c) the waveguide beams are still attached after five minutes of isotropic etching, (d) the array is fully suspended after 17 minutes of isotropic etching

and increased levels of automation. The main result reported in this paper is the fabrication and mechanical characterisation of a variety of flexible waveguide designs.

Single waveguides and waveguide arrays with two and four coupled parallel beams of different lengths $(250 \mu \mathrm{m}$, $500 \mu \mathrm{m}, 750 \mu \mathrm{m}$ and $1000 \mu \mathrm{m})$ and different widths $(18 \mu \mathrm{m}, 26 \mu \mathrm{m}$ and $34 \mu \mathrm{m})$ are designed and fabricated. The measured dimensions are found to be $1 \%$ to $14 \%$ smaller than designed. Single cantilever beams with a width of $14 \mu \mathrm{m}$ and thickness of $15.1 \mu \mathrm{m}$ snap off when the length exceeds approximately $400 \mu \mathrm{m}$. The fabricated devices show a beam curvature resulting in a free-end deflection of $20 \mu \mathrm{m}$ for a length of $988 \mu \mathrm{m}, 10 \mu \mathrm{m}$ for a length of $743 \mu \mathrm{m}, 3 \mu \mathrm{m}$ for a length of $498 \mu \mathrm{m}$ and $0 \mu \mathrm{m}$ for a length of $247 \mu \mathrm{m}$. The curvature has a constant radius of $18.6 \mathrm{~mm}$ and leads to a rotational misalignment of up to $2.7^{\circ}$ for the longest $(988 \mu \mathrm{m})$ and most compliant beams. The beam stiffness of the fabricated devices is measured to be $6.94 \mathrm{~N} \mathrm{~m}^{-1}$ for a length of $498 \mu \mathrm{m}, 2.04 \mathrm{~N} \mathrm{~m}^{-1}$ for a length of $743 \mu \mathrm{m}$ and $0.92 \mathrm{~N} \mathrm{~m}^{-1}$ for a length of $988 \mu \mathrm{m}$.

These results provide a reference for the subsequent research into microactuators to be used for the fine-alignment. In view of the fracturing of suspended waveguide beams, the underlying principle will be studied more thoroughly. The comprehension of waveguide fracturing will be used to improve the releasing process of waveguide structures.

\section{ACKNOWLEDGMENTS}

The research leading to these results has received funding from the Flex-O-Guides project, STW Generic Technologies for Integrated Photonics (GTIP) programme, grant no. 11355 (flexible waveguide development) and from the European Communitys Seventh Framework Programme FP7/2007-2013 under grant agreement ICT 619267 PHASTFlex. We are thankful to our colleagues at the Delft Institute for Microsystems and Nanoelectronics (Dimes) for the support in microfabrication. 


\section{REFERENCES}

[1] Smit, M., Leijtens, X., Bente, E., Van Der Tol, J., Ambrosius, H., Robbins, D., Wale, M., Grote, N., and Schell, M., "Generic foundry model for InP-based photonics," IET Optoelectron 5(5), 187-194 (2011).

[2] Henneken, V. A., Sassen, W. P., van der Vlist, W., Wien, W. H. A., Tichem, M., and Sarro, P. M., "Two-dimensional fiber positioning and clamping device for product-internal microassembly," J Microelectromech Syst 17(3), 724-734 (2008).

[3] van Gurp, J. F. C., Tichem, M., Staufer, U., and Zhao, J., "Passive photonic alignment with submicrometer repeatability and accuracy," IEEE Transactions On Components Packaging and Manufacturing Technology 3(11), 1971-1979 (2013).

[4] De Labachelerie, M., Kaou, N., Armbruster, V., Jeannot, J. C., Mollier, P., Porte, H., and Devoldère, N., "Micromachined connector for the coupling of optical waveguides and ribbon optical fibers," Sens Actuators A Phys 89(1-2), 36-42 (2001).

[5] Roelkens, G., Van Thourhout, D., and Baets, R., "High efficiency grating coupler between silicon-on-insulator waveguides and perfectly vertical optical fibers," Opt. Lett. 32(11), 1495-1497 (2007).

[6] van Zantvoort, J. H. C., Plukker, S. G. L., Dekkers, E. C. A., Khoe, G. D., Koonen, A. M. J., and de Waardt, H., "Effective pigtailing method for fiber arrays to InP-based photonic integrated circuits," IEEE Trans. Adv. Packag. 31(3), 604-611 (2008).

[7] Doany, F. E., Lee, B. G., Assefa, S., Green, W. M. J., Yang, M., Schow, C. L., Jahnes, C. V., Zhang, S., Singer, J., Kopp, V. I., Kash, J. A., and Vlasov, Y. A., "Multichannel high-bandwidth coupling of ultradense silicon photonic waveguide array to standard-pitch fiber array," J Lightwave Technol 29(4), 475-482 (2011).

[8] Popa, D. O. and Stephanou, H. E., "Micro and mesoscale robotic assembly," J. Manuf. Processes 6(1), 52-71 (2004).

[9] Shakespeare, W. J., Pearson, R., Grenestedt, J. L., Hutapea, P., and Gupta, V., "MEMS integrated submount alignment for optoelectronics," J Lightwave Technol 23(2), 504-509 (2005).

[10] Lu, D., "Recent advances on chip-to-chip optical interconnect," in [Photonics and Optoelectronics Meetings (POEM) 2009 - Optoelectronic Devices and Integration ], 7516, 751600-1-751600-14 (2009).

[11] Morichetti, F., Melloni, A., Martinelli, M., Heideman, R. G., Leinse, A., Geuzebroek, D. H., and Borreman, A., "Box-shaped dielectric waveguides: A new concept in integrated optics?," J Lightwave Technol 25(9), 2579-2589 (2007).

[12] Heideman, R., Leinse, A., Hoving, W., Dekker, R., Geuzebroek, D., Klein, E., Stoffer, R., Roeloffzen, C., Zhuang, L., and Meijerink, A., "Large-scale integrated optics using TriPleX ${ }^{\mathrm{TM}}$ waveguide technology: From UV to IR," in [Photonics Packaging, Integration, and Interconnects IX], 7221, 72210R-1-72210R-15 (2009).

[13] Leinse, A., Heideman, R. G., Klein, E. J., Dekker, R., Roeloffzen, C. G. H., and Marpaung, D. A. I., "TriPleX ${ }^{\mathrm{TM}}$ platform technology for photonic integration: Applications from UV through NIR to IR," in [2011 ICO International Conference on Information Photonics, IP 2011], 1-2 (2011).

[14] Fang, W. and Wickert, J. A., "Determining mean and gradient residual stresses in thin films using micromachined cantilevers," Journal of Micromechanics and Microengineering 6(3), 301 (1996). 